\title{
EXAME MACROSCÓPICO DAS ARTICULAÇÕES DE SUÍNOS ARTRÍTICOS NO ABATEDOURO
}

\section{(Macroscopic analysis of joints from arthritic swines pigs in the abattoir)}

\author{
ALTHAUS, L.K.S. ${ }^{1}$; ALBERTON, G.C. ${ }^{2}$; GUIMARÃES, A. M.S. ${ }^{3}$; FIAMETTI, A. ${ }^{4}$ \\ ${ }^{1}$ Curso de Pós-graduação em Ciências Veterinárias - UFPR; \\ ${ }^{2}$ Departamento de Medicina Veterinária - UFPR; \\ ${ }^{3}$ Curso de Medicina Veterinária - UFPR; \\ ${ }^{4}$ Serviço de Inspeção Federal.
}

\begin{abstract}
RESUMO - Realizou-se um estudo macroscópico das articulações dos membros torácicos e pélvicos e respectivos linfonodos de suínos recém-abatidos em um matadouro localizado em Concórdia SC. incluiu-se no presente estudo todos os suínos que foram desviados da linha de abate, por suspeita de artrite. Dos 23.393 suínos abatidos, 140 (0,5\%) foram desviados para o departamento de inspeção final, por suspeita de artrite. Deste total constatou-se: $70 \%$ de artrite causada pela osteocondrose, $21 \%$ artrite infecciosa, $3 \%$ artrite infecciosa e osteocondrose simultaneamente, $1 \%$ apresentaram artrite traumática, $3 \%$ artrite sem diagnóstico conclusivo e $2 \%$ apresentaram-se com as articulações normais. As artrites causadas pela osteocondrose caracterizaram-se pelo grande volume de líquido sinovial e pelas áreas focais de dobras, abas e úlceras na cartilagem articular. As artrites infecciosas caracterizaram-se pela reação nos linfonodos regionais e pela moderada a acentuada hipertrofia da membrana sinovial. O percentual de artrite causada pela osteocondrose foi superior ao encontrado em estudos anteriores e ressalta a necessidade de um diagnóstico diferencial com as artrites infecciosas a fim de se evitar a condenação desnecessária de carcaças.
\end{abstract}

Palavras-chave: osteocondrose, suíno, artrite, abatedouro.

ABSTRACT - A macroscopic study of thoracic and pelvic limb articulations as well as regional lymph nodes was performed in recently-slaughtered swines at a slaughterhouse located in Concordia, SC, Brasil. A total of 23,393 carcasses were included in the study, $140(0.5 \%)$ being diverted on the discharge line by the Department of Final Inspection as suspicious for arthritis. The analysis of the suspicious carcasses showed that $98(70 \%)$ presented arthritis caused by ostechondrosis, $29(21 \%)$ infectious arthritis, four (3\%) infectious arthritis and ostechondrosis simultaneously, two (1\%) traumatic arthritis, four $(3 \%)$ presented arthritis with uncertain diagnosis and three $(2 \%)$ showed normal articulations. The arthritis caused by the osteochondrosis was diagnosed by the increased volume of sinovial fluid and focal areas of folds, flaps and ulcers in the articular cartilage. The infectious arthritis was diagnosed by an inflammatory process involving regional limph nodes and by moderate to accentuated hypertrophy of the sinovial membrane. The percentage of arthritis caused by osteochondrosis was higher when compared to those found in previous studies. This fact emphasizes the need for a differential diagnosis between infectious arthritis and osteochondrosis in order to avoid the unnecessary condemnation of carcasses at slaughterhouses.

Key-words: osteochondrosis, swine, arthritis, abattoir. 


\section{Introdução}

O índice de condenação de carcaças de suínos por artrite tem aumentado significativamente no Brasil (PEREIRA et al., 1999) e em outros países (TITTIGER e ALEXANDER, 1971; CROSS e EDWARDS, 1981; PRATT, 1986), posicionando esta afecção como uma das principais causas de rejeição de carcaças nos abatedouros de suínos.

Várias pesquisas foram conduzidas com o intuito de se esclarecer a etiologia dessas artrites. Nesses estudos verificou-se que a grande maioria das articulações de suínos com artrite são assépticas (TURNER, 1982; MORES et al., 1991; TURNER et al., 1991; BUTTENSCHON et al., 1995) e muitas delas apresentam lesões que não caracterizam um processo infeccioso (JOHNSTON et al., 1987; TURNER et al., 1991).

Existe a possibilidade de que muitas carcaças de suínos que são condenadas ou destinadas ao aproveitamento condicional por apresentarem artrite poderiam ser aproveitadas sem restrições para o consumo humano. Este desperdício eleva os custos de produção dos frigoríficos e reduz a remuneração dos produtores de suínos. Assim, torna-se justificável a realização de um estudo epidemiológico que esclareça a freqüência e o tipo de artrite que acomete os suínos nos matadouros.

As principais causas de artrite em suínos em idade de abate são, em ordem de incidência: osteocondrose, infecção por Erysipelothrix rhusiopathiae, infecção por Mycoplasma hyosynoviae, infecção por Streptococcus sp. e, por último, as artrites traumáticas (JOHNSTON et al.,1987; TURNER et al., 1991; BUTTENSCHON et al., 1995; ALBERTON et al., 2003).

O presente trabalho teve como objetivo classificar e verificar a freqüência de cada tipo de artrite que acomete os suínos em idade de abate.

\section{Materiais e Métodos}

As amostras foram colhidas no período de janeiro a fevereiro de 2004, no Serviço de Inspeção Federal (S.I.F.) 01, em um matadouro no município de Concórdia - SC.

Foram examinados 140 suínos mestiços, com peso vivo de 70 a 120 quilogramas, abatidos por meio de eletrocussão e posterior sangria. Incluíram-se no estudo todos os animais desviados da linha de abate por suspeita de artrite.

Após a identificação dos animais, as articulações afetadas foram abertas para a visualização e classificação das artrites. Os linfonodos axilares da primeira costela e ilíacos foram retirados da carcaça e analisados macroscopicamente. O líquido sinovial de algumas articulações foi colhido com auxílio de uma seringa para mensuração do volume e verificação da sua coloração.

Após a análise macroscópica, as artrites foram classificadas pela metodologia descrita por JOHNSTON et al. (1987) e ALBERTON (2000): Normal: ausência de reação no linfonodo regional e de alterações em todas as estruturas articulares; Infecciosa: linfonodo regional reativo, moderada a acentuada hipertrofia da membrana sinovial; Osteocondrose: ausência de reação no linfonodo regional, presença de uma ou mais áreas focais de dobras, úlceras ou abas na cartilagem articular, discreta a moderada hipertrofia da membrana sinovial; Traumática: ausência de reação no linfonodo regional, membrana sinovial normal ou discretamente hipertrofiada, ausência de lesões na cartilagem articular, líquido sinovial de coloração avermelhada e discretamente aumentada de volume; Sem diagnóstico conclusivo: ausência de reação no linfonodo regional, discreta a moderada hipertrofia da membrana sinovial, acentuado aumento de volume do líquido sinovial e ausência de lesões na cartilagem articular.

\section{Resultados}

No presente estudo, observou-se que $70 \%$ das artrites eram causadas pela osteocondrose (TABELA 1). Estas artrites não oferecem riscos para o homem, pois são assépticas (TURNER et al., 1991; BUTTENSCHON et al., 1995; ALBERTON et al., 2003). A porcentagem de artrite ocasionada por osteocondrose foi maior que a relatada por JOHNSTON et al. (1987), TURNER 
Exame macroscópico das articulações de suínos artríticos no abatedouro

et al., (1991) e ALBERTON et al., (2000) que encontraram respectivamente, $40 \%, 35,5 \%$ e $50 \%$.

Acredita-se que os padrões irregulares de ossificação endocondral são ocorrências comuns nos suínos, e que a maioria dessas lesões passa despercebida, sendo muitas vezes reabsorvida (DOIGE e WEISSBRODE, 1998). Nesse contexto têm-se afirmado que sua prevalência está relacionada a fatores genéticos e ao manejo a que são submetidos os animais (SOBESTIANSKY et al., 2003). De acordo com GRONDALEN (1974) todos os suínos modernos poderiam ser portadores de lesões de osteocondrose, sendo que nos animais selecionados para um rápido desenvolvimento, algumas dessas lesões evoluem, provocando o desencadeamento de artrites ou fraturas. YTREHUS et al. (2004) não observaram influência do ganho de peso diário e do peso de abate no escore de osteocondrose, mas observaram influência na composição genética dos animais na incidência e na localização das lesões. A osteocondrose tem sido descrita também em javalis (STUKELJ, 2002).

TABELA 1 - CARACTERÍSTICAS MACROSCÓPICAS DAS ESTRUTURAS ARTICULARES E DOS LINFONODOS REGIONAIS DE 140 SUÍNOS DESVIADOS DA LINHA DE ABATE POR SUSPEITA DE ARTRITE CONCÓRDIA, SC - BRASIL.

\begin{tabular}{|c|c|c|c|c|c|c|c|}
\hline \multirow{2}{*}{ DIAGNÓSTICO } & \multirow[b]{2}{*}{$\mathrm{N}$} & \multirow[b]{2}{*}{$\%$} & \multicolumn{2}{|c|}{ LÍQUIDO SINOVIAL } & \multirow{2}{*}{$\begin{array}{c}\text { MEMBRANA } \\
\text { SINOVIAL } \\
\text { Grau de } \\
\text { hipertrofia }\end{array}$} & \multirow{2}{*}{$\begin{array}{l}\text { CARTILAGEM } \\
\text { ARTICULAR }\end{array}$} & \multirow{2}{*}{$\begin{array}{l}\text { LINFONODO } \\
\text { REGIONAL }\end{array}$} \\
\hline & & & $\begin{array}{c}\text { Volume } \\
\text { Médio }(\mathrm{ml})\end{array}$ & Características & & & \\
\hline $\begin{array}{l}\text { Artrite causada } \\
\text { por } \\
\text { osteocondrose }\end{array}$ & 98 & 70 & $\begin{array}{c}23,16 \\
{[6.5-85]}\end{array}$ & $\begin{array}{c}\text { Seroso (30) } \\
\text { Serosanguinolento } \\
(68)\end{array}$ & $\begin{array}{c}\text { Discreta (78) } \\
\text { Moderada (19) } \\
\text { Normal (1) }\end{array}$ & $\begin{array}{c}\text { Dobra (27) } \\
\text { Aba (14) } \\
\text { Úlcera (57) }\end{array}$ & $\begin{array}{c}\text { Não reativo } \\
\text { (98) }\end{array}$ \\
\hline $\begin{array}{c}\text { Artrite } \\
\text { infecciosa }\end{array}$ & 29 & 21 & $\begin{array}{c}13,5 \\
{[3,5-50]}\end{array}$ & $\begin{array}{c}\text { Seroso (9) } \\
\text { Serosanguinolento } \\
(20)\end{array}$ & $\begin{array}{c}\text { Moderada (13) } \\
\text { Acentuada } \\
\text { (16) }\end{array}$ & $\begin{array}{c}\text { Erosão (12) } \\
\text { Pannus (5) } \\
\text { Normal (12) }\end{array}$ & $\begin{array}{l}\text { Reativo } \\
\text { (29) }\end{array}$ \\
\hline $\begin{array}{l}\text { Artrite } \\
\text { traumática }\end{array}$ & 2 & 1 & Não avaliado & $\begin{array}{c}\text { Seroso (1) } \\
\text { Serosanguinolento (1) }\end{array}$ & $\begin{array}{l}\text { Discreta (1) } \\
\text { Moderada (1) }\end{array}$ & $\begin{array}{l}\text { Normal (1) } \\
\text { Erosão (1) }\end{array}$ & $\begin{array}{c}\text { Não reativo } \\
\text { (2) }\end{array}$ \\
\hline $\begin{array}{c}\text { Artrite } \\
\text { infecciosa e } \\
\text { osteocondrose }\end{array}$ & 4 & 3 & Não avaliado & $\begin{array}{c}\text { Serosanguinolento (3) } \\
\text { Seroso (1) }\end{array}$ & $\begin{array}{l}\text { Moderada (1) } \\
\text { Acentuada (3) }\end{array}$ & $\begin{array}{c}\text { Dobra (1) } \\
\text { Aba (1) } \\
\text { Úlcera (2) }\end{array}$ & Reativo (4) \\
\hline Normal & 3 & 2 & Não avaliado & $\begin{array}{c}\text { Seroso (1) } \\
\text { Serosanguinolento (2) }\end{array}$ & Normal (3) & Normal (3) & $\begin{array}{c}\text { Não reativo } \\
\text { (3) }\end{array}$ \\
\hline $\begin{array}{c}\text { Artrite sem } \\
\text { diagnóstico } \\
\text { conclusivo }\end{array}$ & 4 & 3 & Não avaliado & $\begin{array}{c}\text { Serosanguinolento (1) } \\
\text { Seroso (3) }\end{array}$ & $\begin{array}{l}\text { Moderada (2) } \\
\text { Acentuada (2) }\end{array}$ & Normal (4) & $\begin{array}{c}\text { Não reativo } \\
\text { (4) }\end{array}$ \\
\hline
\end{tabular}

TABELA 2 - PERCENTUAL DE ARTICULAÇÕES ACOMETIDAS DE ACORDO COM O TIPO DE ARTRITE ENCONTRADA EM 140 SUÍNOS ARTRIITICOS. CONCÓRDIA, SC - BRASIL.

\begin{tabular}{|c|c|c|c|c|c|}
\hline \multirow{2}{*}{$\begin{array}{l}\text { TIPO DE } \\
\text { ARTRITE }\end{array}$} & \multicolumn{5}{|c|}{ ARTICULAÇÃO AFETADA } \\
\hline & Úmero-radial & Fêmurotibial & Ombro & Coxal & Poliartrite \\
\hline Osteocondrose & 76 & 17 & 7 & - & - \\
\hline Infecciosa & 28 & 54 & 6 & - & - \\
\hline $\begin{array}{l}\text { Infecciosa } \\
\text { osteocondrose }\end{array}$ & - & - & - & - & 100 \\
\hline Traumática & - & 100 & - & - & - \\
\hline Sem dignóstico & 25 & 75 & & & \\
\hline
\end{tabular}


FIGURA 1 - SUPERFÍCIE ARTICULAR DISTAL DO ÚMERO DE SUÍNO COM LESÃO DE OSTEOCONDROSE. OBSERVAR A ÚLCERA NA CARTILAGEM ARTICULAR (SETA).

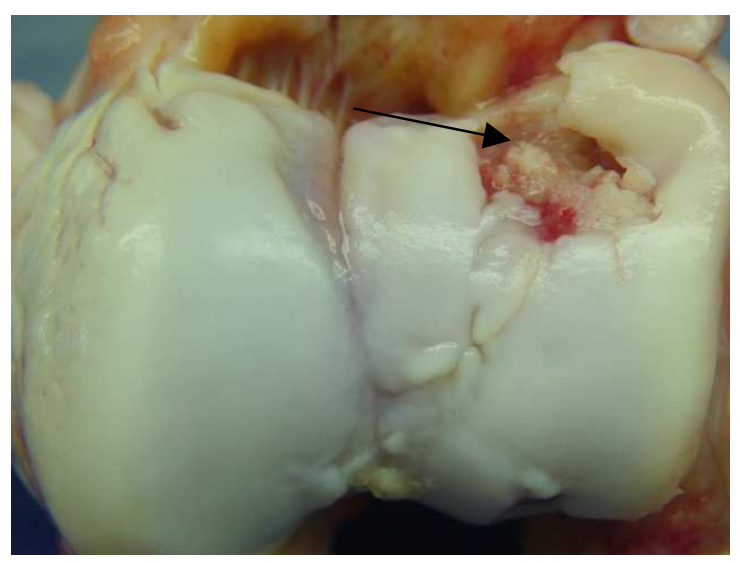

FIGURA 2 - SUPERFÍCIE ARTICULAR DISTAL DO ÚMERO DE SUÍNO COM LESÃO DE OSTEOCONDROSE. OBSERVAR A DOBRA DE CARTILAGEM.

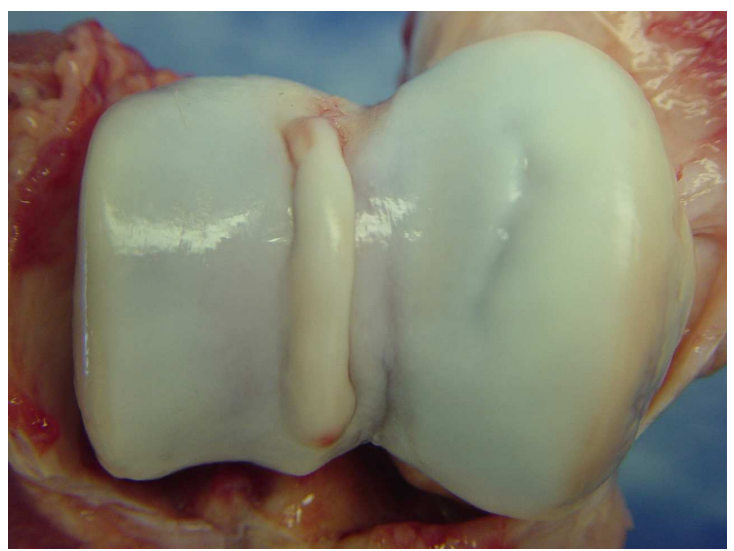

FIGURA 3 - SUPERFÍCIE ARTICULAR DISTAL DO ÚMERO DE SUÍNO COM LESÃO DE OSTEOCONDROSE. OBSERVAR A ABA DE CARTILAGEM.

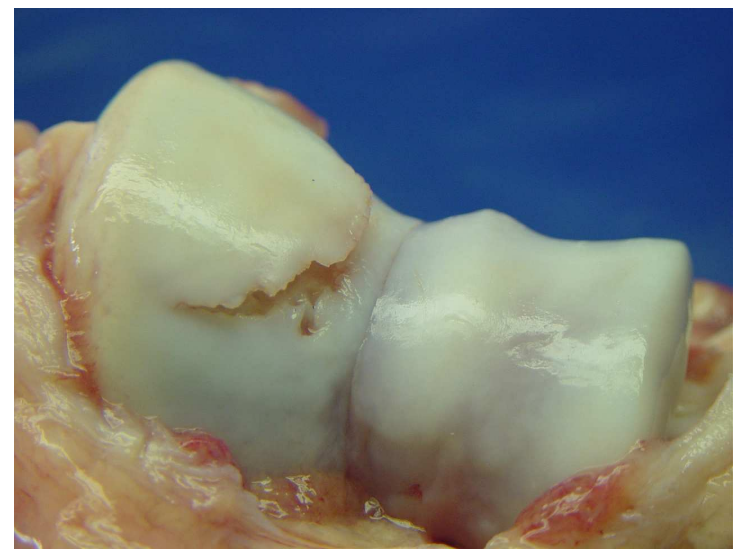



OBSERVAR ACENTUADA HIPERTROFIA DA MEMBRANA SINOVIAL - (SETA).

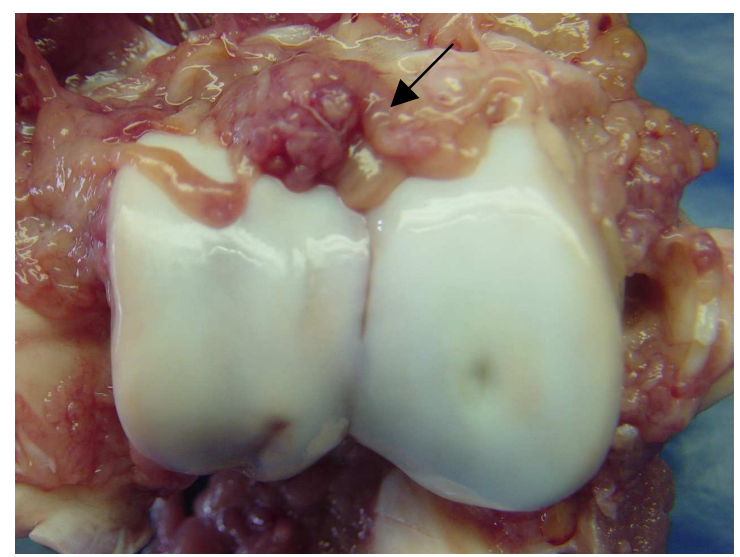

A maioria das pesquisas sobre artrite em suínos não investigou a osteocondrose, limitando-se na maioria das vezes, ao exame microbiológico das articulações. Este fato impossibilita a realização de um estudo retrospectivo visando demonstrar a importância da osteocondrose como causa das artrites em animais de matadouro nas últimas décadas (TURNER, 1982; TURNER et al., 1991; BUTTENSCHON et al., 1995).

Embora os estudos recentes demonstrem que artrite causada pela osteocondrose seja a mais prevalente nos abatedouros, os mapas de inspeção não permitem este estudo retrospectivo, uma vez que nas planilhas todos os tipos de artrite são anotados simplesmente como artrite. Os resultados do presente estudo ressaltam a necessidade de mudança no sistema de coleta de dados do Serviço de Inspeção, de maneira que as artrites causadas por osteocondrose sejam anotadas como osteocondrose. Dessa maneira, os profissionais que atuam na produção dos suínos poderiam instituir medidas de controle adequadas para o tipo de artrite que esteja determinando prejuízos no matadouro.

Tendo em vista que as decisões sanitárias para as carcaças afetadas com artrite infecciosa ou por osteocondrose são diferentes, torna-se necessária à definição de parâmetros que permitam uma diferenciação macroscópica segura. No presente estudo, verificou-se que o melhor parâmetro que possibilita esta diferenciação é a presença ou não de reatividade nos linfonodos regionais. Em todos os casos em que a artrite era de caráter infeccioso foi observada reatividade nos linfonodos. Nos casos de osteocondrose os linfonodos referentes ao membro afetado não apresentaram reatividade. Entretanto deve-se atentar que pode ocorrer simultaneamente osteocondrose e artrite infecciosa nas articulações. Nesses casos a carcaça é condenada prioritariamente como portadora de artrite infecciosa, recebendo o destino estabelecido pelas normas sanitárias.

A membrana sinovial também é um parâmetro de extrema importância, já que nas artrites ocasionadas pela osteocondrose observou-se que em $79 \%$ dos casos essa membrana estava com discreta hipertrofia e, nas artrites infecciosas, $55 \%$ dos casos apresentaram discreta hipertrofia e os demais hipertrofia moderada (FIGURA 4). Essas peculiaridades da membrana sinovial são de suma importância no diagnóstico diferencial entre as artrites infecciosas e não infecciosas. O exame da cartilagem articular também é importante no diagnóstico diferencial, uma vez que a cartilagem com osteocondrose apresenta lesões características como abas, dobras e úlceras (FIGURA 1, 2, 3).

As articulações mais afetadas pela osteocondrose foram as úmero-ulnar (76\%) corroborando os estudo de CROSS e EDWARDS (1981), ao observarem que 
geralmente as artrites causadas pela osteocondrose se localizam nos membros torácicos (TABELA 2).

Aliado ao exame dos linfonodos, da membrana sinovial e da cartilagem articular, o exame do volume e aspecto do líquido sinovial deve ser analisado com cautela no estabelecimento do diagnostico diferencial, pois as artrites causadas pela osteocondrose apresentam um volume muito superior ao das infecciosas e, este líquido geralmente está serosanguinolento. Isto pode influenciar os inspetores a considerar o caso como infeccioso, pelo aspecto repugnante que este líquido apresenta. O volume máximo de líquido sinovial encontrado neste trabalho nas articulações com osteocondrose foi de 85 mililitros, com média de 23,16 mililitros. JONHSTON et al., (1987) e ALBERTON et al. (2003) encontraram médias de 6,0 e $20,25 \mathrm{ml}$, respectivamente.

A freqüência de artrite infecciosa foi de $21 \%$. Embora essa freqüência tenha sido muito inferior ao da osteocondrose, ela merece destaque, uma vez que as carcaças acometidas são destinadas para produto cozido, banha ou subproduto. A porcentagem de artrite infecciosa encontrada nesse trabalho foi maior que a relatada por TURNER et al. (1991) que encontraram $6,1 \%$, e menor que a relatada por JONHSTON et al. (1987), que diagnosticaram $40 \%$ de artrite infecciosa em 52 articulações estudadas.

As articulações mais afetadas pelas artrites infecciosas foram as articulações fêmurotibial $(57 \%)$ o que está de acordo com o observado por CROSS e EDWARDS, (1981). Deste modo, a localização da artrite na carcaça também auxilia no diagnóstico diferencial, uma vez que as artrites causadas pela osteocondrose geralmente se localizam nos membros torácicos e as infecciosas nos membros pélvicos.

As artrites consideradas sem diagnósticos, são aquelas que não se enquadram nem como osteocondrose e nem como artrite infecciosa, uma vez que não apresentam lesões típicas de osteocondrose, apresentando uma hipertrofia moderada a acentuada da membrana sinovial e não possuem reação nos linfonodos. Este tipo de artrite também foi relatado por outros autores; entretanto nenhuma hipótese sobre a gênese destas afecções foi proposta (JOHNSTON et al., 1987; ALBERTON et al., 2000).

A inspeção dos linfonodos ilíacos, devido ao fácil acesso, é feita na linha do abate, possibilitando um diagnóstico prévio de artrite nos membros pélvicos. Em se tratando dos membros torácicos esse prévio diagnóstico é feito apenas na observação do aumento de volume nas articulações que por sua vez são perfuradas na linha de inspeção. $O$ fato do linfonodo axilar da primeira costela situar-se sob a costela dificulta a sua inspeção obrigatória, uma vez que se faz necessário à remoção do esterno para o acesso ao linfonodo em questão, sendo feita apenas pelo Departamento de Inspeção em caso de suspeitas de artrites. Todavia, em alguns frigoríficos, onde as carcaças são serradas antes de passarem pela linha de inspeção, o exame deste linfonodo é facilitado e deveria ser incluso na rotina da linha de inspeção.

\section{Conclusões}

- A principal causa de artrite em suínos em idade de abate é a osteocondrose;

- O percentual de artrites causadas por osteocondrose aumentou significativamente nos últimos anos;

- As artrites infecciosas e não infecciosas podem ser facilmente diferenciadas pelo exame dos linfonodos referentes e das estruturas articulares;

- A inspeção dos linfonodos axilares da primeira costela deve ser incluída na rotina da inspeção de abate de suínos.

\section{REFERÊNCIAS}

ALBERTON, G.C. Estudo anatomopatológico, microbiológico, citológico e físico-químico das articulações de suínos com artrite no abatedouro.Botucatu, 2000. 31p. Tese (Doutorado) - Universidade estadual Paulista "Júlio de Mesquita filho".

ALBERTON, G.C.; BANDARRA, E.P.; PIFFER, I.; MORES, M.A.Z.; PEREIRA, M.A.C; YAMAMOTO, M.T. Exame anatomopatológico, microbiológico, citológico e físico-químico das articulações de suínos artríticos no matadouro. Archives of Veterinary Science, Curitiba, v.8, n.1, p.81-91, 2003. 
ALBERTON, G.C.; PEREIRA, M.A.; YAMAMMOTO, M.T.; BANDARRA, E.P.; SALVO, L.S. Osteocondrose principal causa de artrite em suínos de abatedouro no Brasil. Arquivos de Ciência Veterinárias e Zoologia da UNIPAR, Umuarama, v.3, n.1, p.55-60, 2000.

BUTTENSCHON, J.; SVENSMARK, B.; KYRVAL, J. Non purulent arthritis in Danish slaughter pigs. A study of field cases. Journal of Veterinary Medicine, Verlog, v.42, n.10, p.633-641, 1995.

CROSS, G.M.; EDWARDS, M.J. The detection of arthritis in an abattoir and its public health significance. Australian Veterinary Journal, Artaman, v.57, p.153-158, 1981.

DOIGE, C.E; WEISSBRODE, S.E. Doenças dos ossos e das articulações. In: CARLTON, W.W.; MC GAVIN, M.D. Patologia veterinária especial. 2. ed. PortoAlegre: Armed, 1998. p.474-485.

GRONDALEN, T. Osteochondrosis and arthrosis in pigs. I. Incidence in animal up to $120 \mathrm{Kg}$ live weight. Acta Veterinaria Scandinavica, Oslo, v.15, p.1-25, 1974.

JOHNSTON, K.M.; DOIGE, C.E; OSBORNE, A.D. An evaluation of nonsuppurative joint disease in slaughter pigs. Canadian Veterinary Journal, Ottawa, v.28, n.4. p.174-180, 1987.

MORES, N.; BRITO, M.A.V.P.; YAMAMOTO, M.T.; PEREIRA, M.A.C. Poliartrite em suínos de abatedouro: resultados preliminares das causas e características patológicas. In: CONGRESSO BRASILEIRO DE VETERINÁRIOS ESPECIALISTAS EM SUÍNOS, 5., 1991, Águas de Lindóia. Anais... Águas de Lindóia, 1991. p.85.

PEREIRA, M.A.C.; YAMAMOTO, M.A.; ALBERTON, G.C.; BANDARRA, E.P. Estudo retrospectivo sobre condenação de carcaças de suínos por artrite. In: CONGRESSO BRASILEIRO DE VETERINÁRIOS ESPECIALISTAS EM SUÍNOS, 9., 1999, Belo Horizonte. Anais... Belo Horizonte: ABRAVES, 1999. p.255-256.
PRATT, J.H. Monitoring for health. In: Report on pig health scheme. Annual veterinary conference, 1985, Stoneleigh, London: Ministry of Agriculture, 1986. p.86-95.

SOBESTIANSKY, J.; SOUZA M.A.; LINHARES, D.C.L; MEYER, F. Osteocondrose da Ulna: características clínicas e anatomopatológicas do primeiro caso diagnosticado no Brasil - Relato de caso. In: CONGRESSO BRASILEIRO DE VETERINÁRIOS ESPECIALISTAS EM SUÍNOS, 11, 2003, Goiânia. Anais...Goiânia: ABRAVES, 2003. p.129-130.

STUKELJ, M. A comparison of constitutional features and pathomorphological changes of the foot in wild boar and domestic swine. Veterinarske Novice, v.28, n.10, p. 420-423, 2002.

TITTIGER, F.; ALEXANDER, D.C. Studies on the bacterial flora of condemnedportions from arthritic hogs. Canadian Journal of Comparative Medicine, Ottawa, v.35, p.244248, 1971.

TURNER, G.V.S. A microbiological study of polyarthritis in slaughter pigs. Journal of the South African Veterinary Association, Pretoria, v.53, n.2, p.99-101, 1982.

TURNER, G.V.S.; COLLETT, M.G.; VEARY, C.M.; KRUGER, C. Arthritis in slaughter pigs. Journal of the South African Veterinary Association, v.62, n.3, p.107-109, 1991.

YTREHUS, B; GRINDFLEK, E.; TEIGE, J.; STUBSJOEN, E.; GRONDALEN, T.; CARLSON. C. S. The effect of parentage on the prevalence, severity and location of lesions of osteochondrosis in swine. Journal of Veterinary Medicine Series A, Berlin, v.51, n.4, p.188-195, 2004.

Recebido para publicação: 30/01/2005 Aprovado: 11/04/2005 